Check for updates

Cite this: RSC Adv., 2018, 8, 954

Received 14th October 2017 Accepted 12th December 2017

DOI: $10.1039 / \mathrm{c} 7 \mathrm{ra11325k}$

rsc.li/rsc-advances

\section{Low efficiency roll-off and high color stability pure fluorescent white organic light-emitting diode based exciplex host}

\begin{abstract}
Heng Zhang, $\dagger^{\mathrm{ab}}$ Ziqi Wang, $\dagger^{\mathrm{ab}}$ Long Gao, ${ }^{\mathrm{ab}}$ Bo Zhao (D) *ab and Wenlian Lic
Pure fluorescent WOLEDs with low efficiency roll-off and high color stability were realized by employing an exciplex host. Due to incomplete energy transfer from the blue exciplex host of $\mathrm{MCP}$ : PO-T2T to the orange fluorescent dopant of rubrene, the WOLEDs showed a maximum current efficiency, power efficiency and EQE of $14.2 \mathrm{~cd} \mathrm{~A}^{-1}, 12.8 \mathrm{Im} \mathrm{W}^{-1}$ and $4.90 \%$, respectively. To our surprise, a rather small roll-off ratio of $8.2 \%$ from the maximum EQE to the EQE at $1000 \mathrm{~cd} \mathrm{~m}^{-2}$ and stable white light-emitting spectra with CIE coordinates of $(0.384 \pm 0.001,0.439 \pm 0.002)$ from $4 \mathrm{~V}$ to $8 \mathrm{~V}$ were obtained simultaneously. The bipolarity and triplet exciton up-conversion of the exciplex host played key roles in delivering excellent performance. More detailed discussions are also provided.
\end{abstract}

\section{Introduction}

White organic light-emitting diodes (WOLEDs) have received much attention due to their great potential in solid-state lighting, which is a natural flat-panel source and could be rolled with a flexible substrate. WOLED based phosphorescent materials can achieve almost 100\% internal quantum efficiency (IQE), but high material cost and serious efficiency roll-off still limit their application. The traditional fluorescent materials have the advantages of low cost and high efficiency roll-off; however, their extremely low efficiency limits their development. In recent years, thermally activated delayed fluorescent (TADF) materials have become the focus of research, which could also realize $\sim 100 \%$ IQE because of the efficient upconversion of triplet excitons. ${ }^{\mathbf{1 - 4}}$ However, the efficiency rolloff at high current density still exists, which may originate from concentration quenching, relatively slow up-conversion rate and long triplet exciton lifetime. ${ }^{5}$

In addition to the various emitting materials, there is another emitting exciplex, which is similar to the TADF material, which can also show high efficiency due to the upconversion of triplet excitons. Exciplex forms due to the charge transfer between the highest occupied molecular orbital (HOMO) of the donor and the lowest unoccupied molecular

\footnotetext{
${ }^{a}$ Key Laboratory of Interface Science and Engineering in Advanced Materials, Ministry of Education, Taiyuan University of Technology, Taiyuan 030024, P. R. China

${ }^{b}$ Research Center of Advanced Materials Science and Technology, Taiyuan University of Technology, Taiyuan 030024, P. R. China.E-mail: zhaobo01@tyut.edu.cn

${ }^{c}$ State Key Laboratory of Luminescence and Applications, Changchun Institute of Optics, Fine Mechanics, and Physics, Chinese Academy of Sciences, Changchun 130033, P. R. China

$\dagger$ These two authors contributed equally to this work.
}

orbital (LUMO) of the acceptor molecule. Many highly efficient exciplexes with various monochromatic lights have been reported in the past several years. ${ }^{6-11}$ However, large efficiency roll-off could also be observed with exciplex OLEDs. Kim et al. provided an alternative way using an exciplex as the host, and achieving a series of high efficiency phosphorescent OLEDs. ${ }^{\mathbf{1 2 - 1 5}}$ Our group also obtained highly efficient orange and red OLEDs by utilizing the energy transfer from exciplexes to dopants. ${ }^{16,17}$ Compared to exciplexes as emitters, we consider that exciplexes are more suitable as hosts due to the following attributes: (1) the bipolar carriers transport because of the donor and acceptor material, which are the hole and electron transport material, respectively. (2) The broad recombination zone could reduce the excitons' concentration. (3) Low turn-on voltage; the barrier-free carriers' injection from the transport layer to the emitting layer (EML) and the small intrinsic emitting band gap of exciplex could lower the voltage. In particular, the low turn-on voltage could improve the power efficiency greatly, which is a more important parameter in WOLEDs. Therefore, exciplex hosts have great potential application in WOLEDs, but most WOLEDs with exciplex as the host reported to date were based on phosphorescent materials, ${ }^{18-20}$ which have the problems of high cost and resource shortage. Hence, we attempted to obtain the pure fluorescent WOLEDs with high efficiency and low efficiency rolloff by utilizing an exciplex as the host.

In this study, we designed the pure fluorescent WOLEDs with a very simple structure to achieve low efficiency roll-off using a blue exciplex as the host and an orange traditional fluorescent material, (5,6,11,12)-tetraphenyl-naphthacene (rubrene), as the dopant. The donor and acceptor materials of the blue exciplex are $m$-bis( $N$-carbazolyl)benzene (mCP) and (1,3,5-triazine-2,4,6triyl)-tris(benzene-3,1-diyl)-tris(diphenylphosphine oxide) (PO$\mathrm{T} 2 \mathrm{~T})$, respectively. By the introduction of the dopant rubrene 
in an optimal concentration, highly efficient fluorescent WOLEDs are obtained with maximum current efficiency, power efficiency and external quantum efficiency (EQE) of $14.2 \mathrm{~cd} \mathrm{~A}^{-1}$, $12.8 \mathrm{~lm} \mathrm{~W}^{-1}$ and $4.90 \%$, respectively. In particular, the EQE rolloff ratio is only $8.2 \%$ from the maximum EQE to the EQE at 1000 cd $\mathrm{m}^{-2}$. Simultaneously, the Commission Internationale de I'Eclairage (CIE) coordinates of $(0.384,0.440)$ are obtained at $6 \mathrm{~V}$, along with a very small variation of $\pm(0.001,0.002)$ from $4 \mathrm{~V}$ to $8 \mathrm{~V}$. The exciplex host bipolarity, broad recombination zone, high energy transfer efficiency, balanced carriers transport recombination and efficient triplet excitons up-conversion are responsible for the low efficiency roll-off and high color stability of the WOLEDs.

\section{Experimental section}

All the devices were fabricated on indium tin oxide (ITO) coated glass substrates with a sheet resistance of $10 \Omega \mathrm{sq}^{-1}$. The ITO substrates were cleaned with acetone, deionized water, and acetone, in sequence, and then, treated by ultraviolet-ozone for 15 min. Subsequently, the ITO substrates were loaded into a high vacuum chamber (approximately $3 \times 10^{-4} \mathrm{~Pa}$ ) for subsequent deposition. After the deposition of organic-inorganic layers, $\mathrm{Al}$ cathode was deposited in the end with a shadow mask, which defined the device area of $3 \times 3 \mathrm{~mm}^{2}$. The UV-vis absorption spectrum was measured with a Hitachi U-3900 scanning spectrophotometer. The photoluminescence (PL) spectra were measured with a FluoroMax-4 fluorescence spectrometer (HORIBA Jobin Yvon). The electroluminescent (EL) spectra were measured using a PR-655 spectra scan spectrometer with computer control. The current-voltage-luminance characteristics were measured by a measuring system of the Keithley 2400 power supply combined with a BM-7A luminance colorimeter. EQE was calculated from the EL spectra data and current density-voltage-luminance curve. All the materials were procured commercially and used without further purification. All the measurements were carried out at room temperature and under ambient conditions without any protective coatings.

\section{Results and discussion}

First, we studied the EL performance of the exciplex with mCP as the donor and PO-T2T as the acceptor. The structure of the OLEDs is designed as follows: ITO/ $\mathrm{MoO}_{3}(3 \mathrm{~nm}) / \mathrm{mCP}(20 \mathrm{~nm}) /$ mCP : PO-T2T (1 : 1) (15 nm)/PO-T2T (35 nm)/LiF (1 nm)/Al. The structure is very simple with only two organic materials of donor and acceptor, while the mixed ratio of donor and acceptor in EML is $1: 1$. The pure films of $\mathrm{mCP}$ and PO-T2T act as the hole transport layer (HTL) and the electron transport layer (ETL), respectively. Fig. 1 shows the EL performance, including current efficiency, power efficiency, EQE and EL spectra under different voltages. The device exhibits blue light emission with peak value at $\sim 473 \mathrm{~nm}$ and the EL spectra remain the same under different voltages, which demonstrates the intrinsic emission of the exciplex without impurity. The blue exciplex OLEDs also achieve high efficiency with the maximum current efficiency, power efficiency and EQE of $11.1 \mathrm{~cd} \mathrm{~A}^{-1}, 8.5 \mathrm{~lm} \mathrm{~W}^{-1}$ and $6.0 \%$,
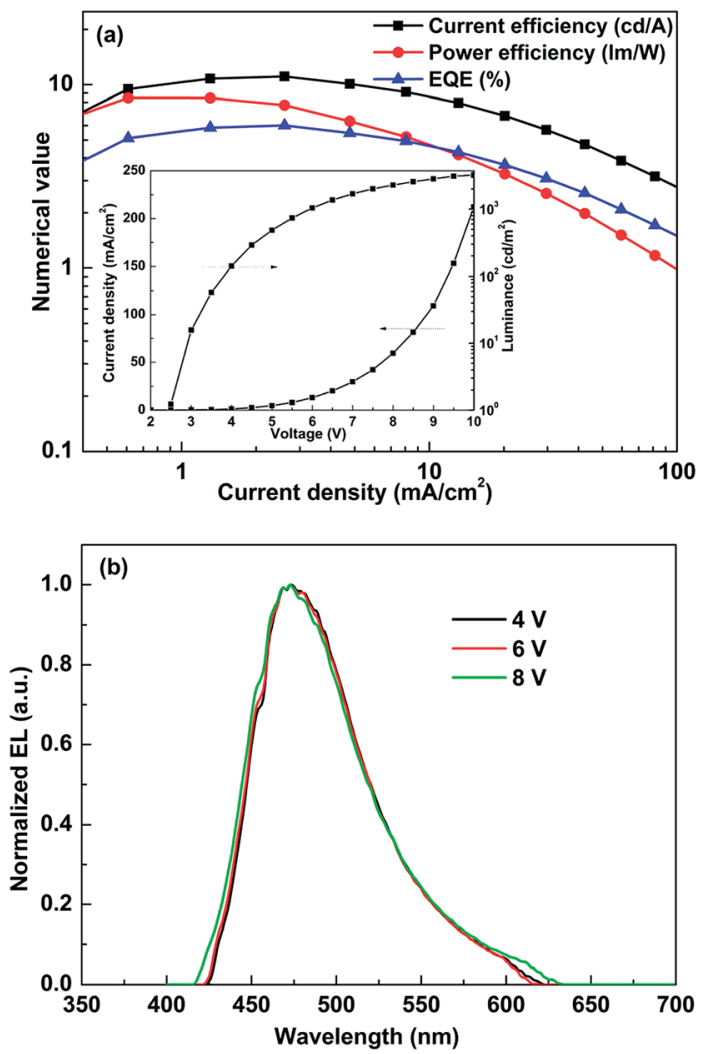

Fig. 1 The EL performance of blue exciplex. (a) Current efficiency, power efficiency and EQE curves. Inset is the current densityvoltage-luminance curve. (b) The EL spectra under different voltages.

respectively. The efficiency level is comparable to previous reports, ${ }^{\mathbf{2 1 , 2 2}}$ which derive from the balanced recombination of holes and electrons, and the triplet excitons up-conversion also contributes to the excellent device performance.

The fluorescent WOLEDs could be realized by introducing an orange dopant of rubrene based on the blue exciplex OLEDs. Therefore, the energy transfer efficiency between the exciplex host and the dopant plays a key role in highly efficient WOLEDs. In general, the degree of overlapping between the emitting spectrum of the host and the absorption spectrum of the dopant could represent the energy transfer efficiency. ${ }^{23,24}$ The PL spectrum of the exciplex and the absorption spectrum of rubrene ${ }^{\mathbf{1 6}}$ are displayed in Fig. 2. It is evident that a large overlap exists between the PL spectrum of the blue exciplex and the absorption spectrum of the dopant, which indicates high energy transfer efficiency from the blue exciplex to rubrene.

The WOLEDs with the simple structure based on the blue exciplex OLED are designed as follows: ITO/ $\mathrm{MoO}_{3}(3 \mathrm{~nm}) / \mathrm{mCP}$ $(20 \mathrm{~nm}) / \mathrm{mCP}:$ PO-T2T $(1: 1): x \mathrm{wt} \%$ rubrene $(15 \mathrm{~nm}) / \mathrm{PO}-\mathrm{T} 2 \mathrm{~T}$ $(35 \mathrm{~nm}) / \mathrm{LiF}(1 \mathrm{~nm}) / \mathrm{Al}$ (where the $x=0.8,1.6$ and 2.4 , respectively). Fig. 3 shows the device structure and EL performance of the WOLEDs with different concentrations. All the WOLEDs exhibit very low turn-on voltages of $\sim 2.5 \mathrm{~V}$ and high emitting luminance of $>10000 \mathrm{~cd} \mathrm{~m}^{-2}$. The low turn-on voltage and high emitting luminance indicate the high recombination efficiency in the EML. ${ }^{25}$ The almost same current density-voltage $(J-V)$ 


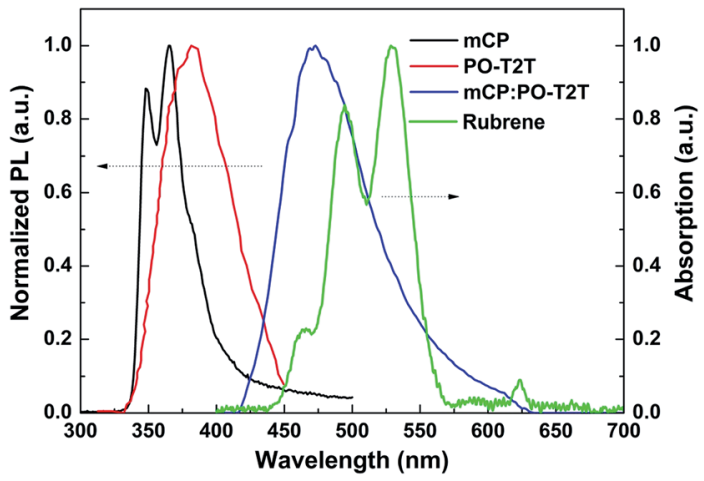

Fig. 2 The PL spectra of $m C P, P O-T 2 T$ (solution), $m C P$ : PO-T2T exciplex and absorption spectrum of rubrene dopant.

curves under different concentrations demonstrate that the emission of the dopant primarily originates due to the energy transfer from the exciplex host. ${ }^{26,27}$ At the concentration of $1.6 \mathrm{wt} \%$, the WOLEDs achieve the best EL performance with the maximum current efficiency, power efficiency and EQE of 14.2 $\mathrm{cd} \mathrm{A}^{-1}, 12.8 \mathrm{~lm} \mathrm{~W}^{-1}$ and $4.90 \%$, respectively. The EQE of $4.90 \%$ nearly reaches the upper limit of fluorescent quantum efficiency of $5 \%$. The other two WOLEDs with concentrations of $0.8 \mathrm{wt} \%$ and $2.4 \mathrm{wt} \%$ also realize a high maximum EQE of $4.45 \%$ and $4.02 \%$, respectively. The lower concentration of $0.8 \mathrm{wt} \%$ reduces the Förster energy transfer efficiency from the exciplex host to the dopant, and a higher concentration of $2.4 \mathrm{wt} \%$ results in the occurrence of an adverse Dexter energy transfer process between the triplet energy level of exciplex host and dopant.
Hence, the dopant concentration in this structure plays a key role and the lower and higher concentration would both influence the device efficiency.

Table 1 lists the EL performance of the WOLEDs and the rolloff ratio from the maximum EQE to the EQE at $1000 \mathrm{~cd} \mathrm{~m}^{-2}$. All the WOLEDs with different concentrations exhibit a warm white light emission with the CIE coordinates of $(0.368,0.433),(0.384$, $0.440)$ and $(0.440,0.474)$ at $6 \mathrm{~V}$. More surprising is the efficiency roll-off of the WOLEDs. All the three WOLEDs possess a rather low roll-off ratio of just $7.9 \%, 8.2 \%$ and $6.7 \%$ from the maximum EQE to the EQE at $1000 \mathrm{~cd} \mathrm{~m}^{-2}$. In general, the rolloff at high current density derives from the singlet-triplet annihilation (STA), ${ }^{28,29}$ triplet-triplet annihilation (TTA) ${ }^{30}$ and triplet-polaron annihilation (TPA). ${ }^{31}$ In our devices with an exciplex as the host, the broad excitons recombination zone due to the bipolar carriers transport could efficiently reduce the excitons' concentration, which could prevent the STA and TTA. Moreover, the barrier-free carriers' injection from the transport layer to the EML could eliminate charge accumulation and suppress the charge trap, which contribute to decreasing the TPA. However, the blue exciplex device based on the above two points should also have a low roll-off, but unfortunately, the roll-off ratio reaches $\sim 26 \%$ with the blue exciplex device. Hence, there must exist a key process that could efficiently reduce the roll-off. We could notice that the exciplex of MCP : PO-T2T conducts a very small singlet-triplet energy level gap $\left(\Delta E_{\mathrm{ST}}\right)$ and the singlet excited state energy is almost the same with triplet excited state energy $\left(S_{1} \approx T_{1}=2.64 \mathrm{eV}\right)$ from the fluorescent and phosphorescent spectra. ${ }^{22}$ Hence, the upconversion process may be realized; further, considering the (a)

\begin{tabular}{|c|}
\hline LiF (1 nm)/AI \\
\hline PO-T2T (35 nm) \\
\hline mCP:PO-T2T (1:1): \\
x wt\% Rubrene (15 nm) \\
\hline $\mathrm{mCP}^{2}(20 \mathrm{~nm})$ \\
\hline $\mathrm{MoO}_{3}(3 \mathrm{~nm})$ \\
\hline ITO \\
\hline
\end{tabular}

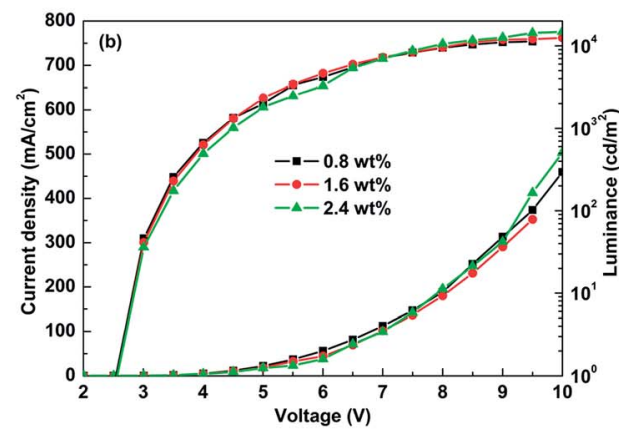



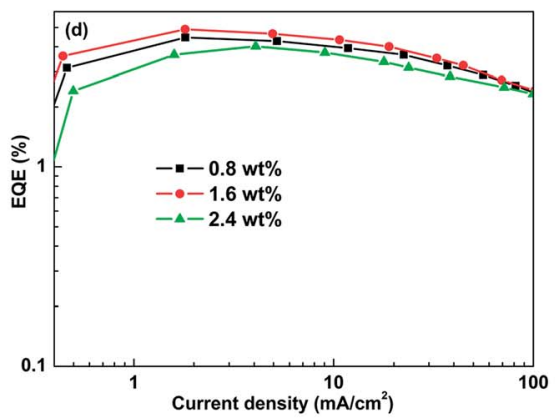

Fig. 3 The device structure and EL performance of the WOLEDs with different concentrations. (a) The device structure. (b) Current densityvoltage-luminance curves. (c) Current efficiency-current density-power efficiency curves. (d) The EQE curves. 
Table 1 Summary EL data and roll-off ratio of all the WOLEDs in this paper

\begin{tabular}{|c|c|c|c|c|c|}
\hline $\begin{array}{l}x \text { wt } \% \\
\text { rubrene }\end{array}$ & $\begin{array}{l}V_{\mathrm{on}}{ }^{a} \\
{[\mathrm{~V}]}\end{array}$ & $\begin{array}{l}\eta_{\mathrm{c}, \max .} / \eta_{\mathrm{p}, \text { max. }} / \mathrm{EQE}_{\max .}{ }^{b} \\
{\left[\mathrm{~cd} \mathrm{~A}^{-1} / \mathrm{lm} \mathrm{W}^{-1} / \%\right]}\end{array}$ & $\begin{array}{l}\eta_{\mathrm{c}, 1000} / \eta_{\mathrm{p}, 1000} / \mathrm{EQE}_{1000}{ }^{c} \\
{\left[\mathrm{~cd} \mathrm{~A}^{-1} / \mathrm{lm} \mathrm{W}^{-1} / \%\right]}\end{array}$ & $\mathrm{EQE}_{\text {max. }} \sim \mathrm{EQE}_{1000}^{d}[\%]$ & $\begin{array}{l}\text { CIE coordinates } \\
\text { at } 6 \mathrm{~V}\end{array}$ \\
\hline 0.8 wt $\%$ & 2.5 & $12.8 / 11.4 / 4.45$ & $11.7 / 8.7 / 4.10$ & 7.9 & $(0.368,0.433)$ \\
\hline $2.4 \mathrm{wt} \%$ & 2.5 & $12.2 / 9.9 / 4.02$ & $11.4 / 8.0 / 3.75$ & 6.7 & $(0.440,0.474)$ \\
\hline
\end{tabular}

${ }^{a}$ Turn-on voltage (the voltage at $\left.1 \mathrm{~cd} \mathrm{~m}^{-2}\right) .{ }^{b}$ Current efficiency $\left(\eta_{\mathrm{c}}\right)$, power efficiency $\left(\eta_{\mathrm{p}}\right)$ and EQE at maximum. ${ }^{c} \eta_{\mathrm{c}}, \eta_{\mathrm{p}}$ and EQE at $1000 \mathrm{~cd} \mathrm{~m}{ }^{-2}$. ${ }^{d} \mathrm{EQE}$ roll-off ratio from maximum to $1000 \mathrm{~cd} \mathrm{~m}^{-2}$.

highly efficient energy transfer efficiency between the exciplex and rubrene, we suggest that the rapid up-conversion and energy transfer are the main factors for the low roll-off. Because small $\Delta E_{\mathrm{ST}}$ is always followed by small singlet excitons radiative rate, the blue exciplex exhibits a relatively large roll-off due the high singlet and triplet excitons density. ${ }^{29}$ Therefore, the more efficient Förster resonance energy transfer, rather than singlet radiation, is beneficial to the small roll-off ${ }^{32,33}$ and utilizing the exciplex as the host to sensitize the dopant is an effective way to reduce the efficiency roll-off.

Fig. 4 shows the EL spectra of the three WOLEDs under the large voltage range from $4 \mathrm{~V}$ to $8 \mathrm{~V}$. All the three WOLEDs display color stable warm white light-emitting spectra and the spectra are almost the same with increasing voltage. The CIE coordinates change ratio of the three WOLEDs are merely $\pm(0.007,0.003), \pm(0.001,0.002)$ and $\pm(0.017,0.006)$, respectively. Moreover, the blue exciplex emitting intensity decrease gradually as the concentrations increase from $0.8 \mathrm{wt} \%$ to $2.4 \mathrm{wt} \%$, which confirm again the orange emission of rubrene derived from the energy transfer of the exciplex host. We suggest that the balanced transport and recombination of carriers and highly efficient energy transfer efficiency results in balanced emitting intensity of the blue exciplex host and the orange dopant. ${ }^{34}$ The high singlet and triplet excited state
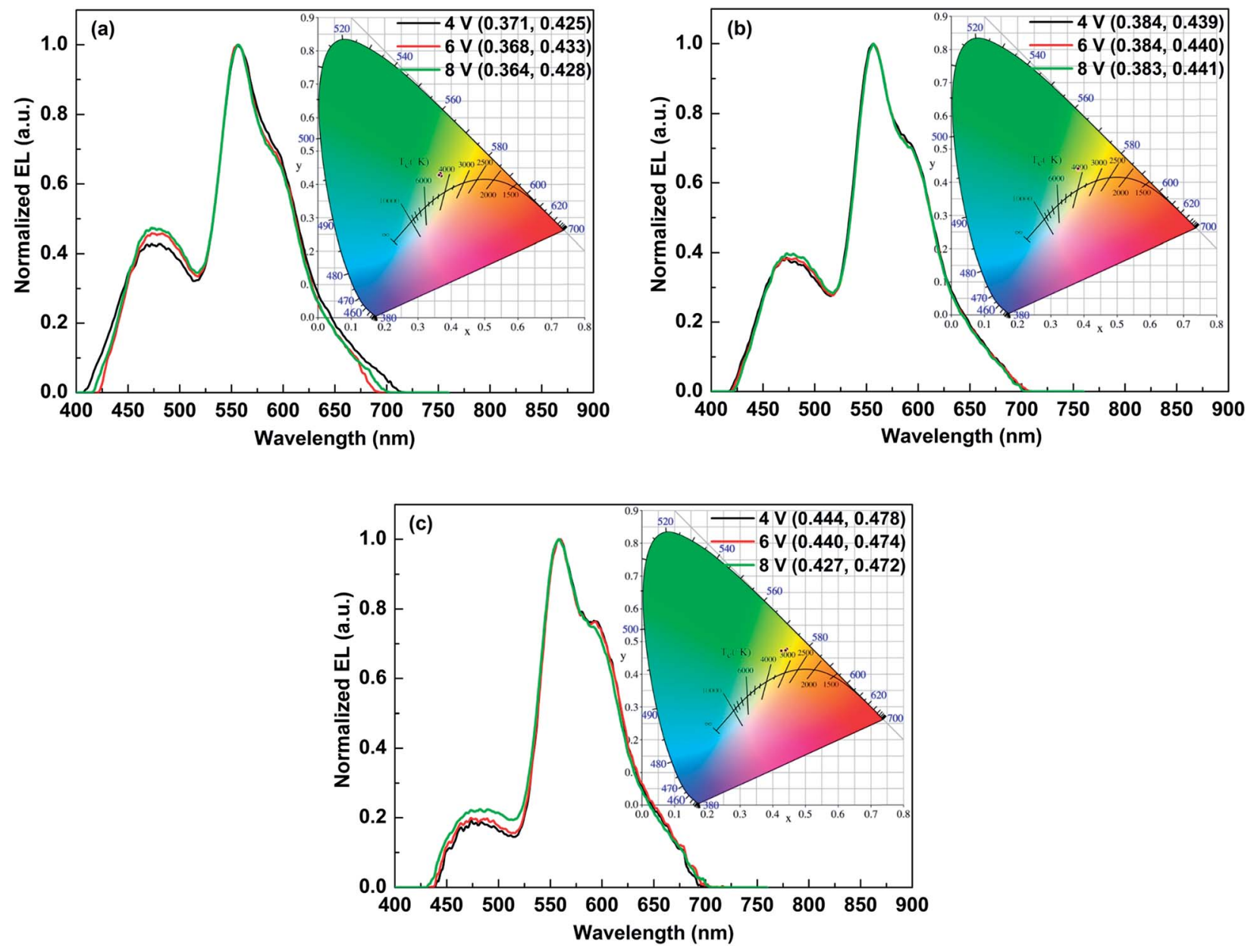

Fig. 4 The EL spectra of the three WOLEDs under different voltages. (a) $0.8 w t \%$. (b) 1.6 wt\%. (c) 2.4 wt\%. 




Fig. 5 The expected energy transfer schematic diagram and EL emission process in this system.

energy of $\mathrm{mCP}$ and PO-T2T transport layer efficiently confine the excitons in EML and stabilize the recombination zone, which also contributes to the color stable white EL spectra.

As mentioned above, the emission of the dopant primarily originated due to the energy transfer from the exciplex host. Finally, we discuss the energy transfer process in this exciplex host-guest system. Fig. 5 shows the expected energy transfer schematic diagram and the EL emission process in this system. The donor of mCP and the acceptor of PO-T2T form the exciplex with a similar singlet and triplet energy level, which could achieve the efficient up-conversion of exciplex triplet excitons. In addition, the higher triplet excitons energy of $\operatorname{mCP}\left(T_{1}=2.94\right.$ $\mathrm{eV})$ and PO-T2T $\left(T_{1}=2.99 \mathrm{eV}\right)$ could efficiently confine the exciplex excitons $\left(S_{1} \approx T_{1}=2.64 \mathrm{eV}\right)$ in EML. The white light emission could be obtained through the incomplete Förster resonance energy transfer process from the exciplex host to the dopant controlling the concentration, which would result in the emission of the blue exciplex host and the orange dopant rubrene simultaneously. Moreover, the invalid Dexter energy transfer process between the triplet energy level of the exciplex host and the dopant could be avoided as far as possible by optimizing the concentration. Although the obtained efficiency in this study is not very good, maybe stemming from the occurrence of Dexter energy transfer and low radiative efficiency of rubrene, the efficiency roll-off are improved with exciplex as the host due to the decreased excitons density with the efficient energy transfer of Förster and Dexter process.

\section{Conclusion}

In conclusion, low efficiency roll-off and high color stability pure fluorescent WOLEDs are realized successfully by utilizing a blue exciplex as the host and orange rubrene as the dopant. With the optimal concentration of $1.6 \mathrm{wt} \%$, the WOLEDs achieve a high current efficiency, power efficiency and EQE of 14.2 $\mathrm{cd} \mathrm{A}^{-1}, 12.8 \mathrm{~lm} \mathrm{~W}{ }^{-1}$ and $4.90 \%$, respectively. Moreover, a rather small roll-off ratio of $8.2 \%$ from the maximum EQE to the EQE at $1000 \mathrm{~cd} \mathrm{~m}^{-2}$ and extremely small variation of CIE coordinates with $\pm(0.001,0.002)$ from $4 \mathrm{~V}$ to $8 \mathrm{~V}$ are also obtained. The exciplex host's bipolarity, broad recombination zone, high energy-transfer efficiency, balanced carriers transport recombination and exciplex host triplet excitons up-conversion are the reasons for low efficiency roll-off and high color stability with the WOLEDs. Finally, we provide a simple way of exciplex host to improve the efficiency roll-off and the higher efficiency would be developed in the future studies.

\section{Conflicts of interest}

There are no conflicts to declare.

\section{Acknowledgements}

This work was financially supported by the National Natural Science Foundation of China (Grant No. 61605137, 61605138, 61705156, 61775155 and 61704118); Scientific and Technological Innovation Programs of Higher Education Institutions in Shanxi (STIP, Grant No. 2016134).

\section{Notes and references}

1 H. Uoyama, K. Goushi, K. Shizu, H. Nomura and C. Adachi, Nature, 2012, 492, 234.

2 S. Hirata, Y. Sakai, K. Masui, H. Tanaka, S. Y. Lee, H. Nomura, N. Nakamura, M. Yasumatsu, H. Nakanotani, Q. Zhang, K. Shizu, H. Miyazaki and C. Adachi, Nat. Mater., 2015, 14, 330.

3 Q. Zhang, B. Li, S. Huang, H. Nomura, H. Tanaka and C. Adachi, Nat. Photonics, 2014, 8, 326.

4 Y. Kitamoto, T. Namikawa, T. Suzuki, Y. Miyata, H. Kita, T. Sato and S. Oi, Org. Electron., 2016, 34, 208-217.

5 J. W. Sun, K. H. Kim, C. K. Moon, J. H. Lee and J. J. Kim, ACS Appl. Mater. Interfaces, 2016, 8, 9806-9810.

6 K. Goushi, K. Yoshida, K. Sato and C. Adachi, Nat. Photonics, 2012, 6, 253-258.

7 K. Goushi and C. Adachi, Appl. Phys. Lett., 2012, 101, 023306.

8 Y. S. Park, K. H. Kim and J. J. Kim, Appl. Phys. Lett., 2013, 102, 153306.

9 J. Li, H. Nomura, H. Miyazaki and C. Adachi, Chem. Commun., 2014, 50, 6174-6176.

10 Y. Zhao, L. Duan, X. Zhang, D. Q. Zhang, J. Qiao, G. F. Dong, L. D. Wang and Y. Qiu, RSC Adv., 2013, 3, 21453-21460.

11 D. C. Chen, Z. H. Wang, D. Wang, Y. C. Wu, C. C. Lo, A. Lien, Y. Cao and S. J. Su, Org. Electron., 2015, 25, 79-84.

12 Y. S. Park, S. Lee, K. H. Kim, S. Y. Kim, J. H. Lee and J. J. Kim, Adv. Funct. Mater., 2013, 23, 4914-4920.

13 S. Lee, K. H. Kim, D. Limbach, Y. S. Park and J. J. Kim, Adv. Funct. Mater., 2013, 23, 4105-4110.

14 H. Shin, S. Lee, K. H. Kim, C. K. Moon, S. J. Yoo, J. H. Lee and J. J. Kim, Adv. Mater., 2014, 26, 4730-4734.

15 S. Lee, H. Shin and J. J. Kim, Adv. Mater., 2016, 26, 58645868.

16 B. Zhao, Y. Q. Miao, Z. Q. Wang, W. H. Chen, K. X. Wang, H. Wang, Y. Y. Hao, B. S. Xu and W. L. Li, Org. Electron., 2016, 37, 1-5.

17 B. Zhao, T. Y. Zhang, B. Chu, W. L. Li, Z. S. Su, H. R. Wu, X. W. Yan, F. M. Jin, Y. Gao and C. Y. Liu, Sci. Rep., 2015, 5, 10697.

18 Z. Chen, X. K. Liu, C. J. Zheng, J. Ye, C. L. Liu, F. Li, X. M. Ou, C. S. Lee and X. H. Zhang, Chem. Mater., 2015, 27, 52065211.

19 X. K. Liu, Z. Chen, J. Qing, W. J. Zhang, B. Wu, H. L. Tam, F. R. Zhu, X. H. Zhang and C. S. Lee, Adv. Mater., 2015, 27, 7079-7085. 
20 D. D. Zhang, L. Duan, Y. G. Zhang, M. H. Cai, D. Q. Zhang and Y. Qiu, Light: Sci. Appl., 2015, 4, e232.

21 W. Y. Hung, G. C. Fang, S. W. Lin, S. H. Cheng, K. T. Wong, T. Y. Kuo and P. T. Chou, Sci. Rep., 2014, 4, 5161.

22 J. H. Lee, S. H. Cheng, S. J. Yoo, H. Shin, J. H. Chang, C. I. Wu, K. T. Wong and J. J. Kim, Adv. Funct. Mater., 2015, 25, 361-366.

23 T. Förster, Ann. Phys., 1948, 437, 55-75.

24 D. L. Dexter, J. Chem. Phys., 1953, 21, 863.

25 J. R. Koo, S. J. Lee, G. W. Hyung, D. W. Im, H. S. Yu, J. H. Park, K. H. Lee, S. S. Yoon, W. Y. Kim and Y. K. Kim, AIP Adv., 2012, 2, 012117.

26 M. Uchida, C. Adachi, T. Koyama and Y. Taniguchi, J. Appl. Phys., 1999, 86, 1680-1687.
27 J. S. Chen and D. G. Ma, J. Appl. Phys., 2004, 95, 5778-5781. 28 Y. Zhang and S. R. Forrest, Phys. Rev. Lett., 2014, 113, 189903. 29 C. Li, L. Duan, D. Zhang and Y. Qiu, ACS Appl. Mater. Interfaces, 2015, 7, 15154-15159.

30 S. P. Wang, Y. W. Zhang, W. P. Chen, J. B. Wei, Y. Liu and Y. Wang, Chem. Commun., 2015, 51, 11972-11975.

31 C. Murawski, K. Leo and M. C. Gather, Adv. Mater., 2013, 25, 6801-6827.

32 D. D. Zhang, M. H. Cai, Y. G. Zhang, Z. Y. Bin, D. Q. Zhang and L. Duan, ACS Appl. Mater. Interfaces, 2016, 8, 3825-3832.

33 H. Fukagawa, T. Shimizu, T. Kamada, S. Yui, M. Hasegawa, K. Morii and T. Yamamoto, Sci. Rep., 2015, 5, 9855.

34 Y. S. Park, J. W. Kang, D. M. Kang, J. W. Park, Y. H. Kim, S. K. Kwon and J. J. Kim, Adv. Mater., 2008, 20, 1957-1961. 